\title{
Diretrizes para elaboração de um protocolo e relatório de estudo de degradação forçada de medicamentos de acordo com a RDC 53/2015
}

\author{
Guidelines of forced degradation studies protocol and report of \\ drug products according to RDC 53/2015
}

Recebido em: 15/03/2018

Aceito em: 21/09/2018

\author{
Gabriel Nunes da $\operatorname{COSTA}^{1}$; Gil Mendes VIANA ${ }^{2}$; Ubiracir Fernandes LIMA \\ FILHO $^{2}$; Lucio Mendes CABRAL ${ }^{2}$ \\ ${ }^{1}$ Departamento de Assuntos Regulatórios, GlaxoSmithKline do Brasil Ltda. \\ Estrada dos Bandeirantes 8464, Jacarepaguá, CEP 22783-110. Rio de Janeiro, \\ RJ, Brasil. ${ }^{2}$ Departamento de Fármacos e Medicamentos, Faculdade de Farmácia, \\ Universidade Federal do Rio de Janeiro. Av. Carlos Chagas Filho 373 Prédio do \\ CCS, Bloco L subsolo sala 19, CEP 21941-902. Rio de Janeiro, RJ, Brasil. \\ E-mail:lmcabral@pharma.ufrj.br
}

\section{ABSTRACT}

The forced degradation study (FDS) is a key to predict the stability of an active pharmaceutical ingredient (API) isolated or in the finished product. From this, it is possible to obtain a stability indicating method by performing severe stress conditions able of to quantify all the possible degradants in the API or in the finished product. The international guidelines about this topic only give an overview on how to perform the FDS, it does not provide methodological details on a practical approach of these studies, which makes scientific standardization difficult. With the publication of RDC 53/2015 and guidelines 04/2015 by Anvisa, the international FDS practices should be revised to obtain a drug registration in Brazil. Therefore, the aim of this study is propose a template for FDS protocol and report that meets the requirements of Brazilian legislation. Degradation path, stress conditions, endpoints, percentage of degradation, mass balance, potential degradation profile, analytical validation, identification and qualification of degradation products were extensively discussed throughout this article. The model protocol and report presented complies with RDC $53 / 2015$ in its entirety.

Keywords: degradation product; forced degradation protocol; forced degradation report forced degradation study; stress study.

\section{RESUMO:}

O estudo de degradação forçada (EDF) é uma atividade preditiva chave para conhecer a estabilidade de um insumo farmacêutico ativo (IFA) e do seu respectivo produto acabado. A partir dele, é possível obter um método indicativo de estabilidade pela realização de condições de estresse severas capaz de quantificar todos os degradantes possíveis no IFA ou no seu produto acabado. Os principais guias internacionais que tratam sobre o tema apenas dão uma visão geral sobre como realizar o EDF não fornecendo detalhes sobre uma abordagem prática destes estudos, o que dificulta uma padronização científica da metodologia. Com a publicação da RDC 53/2015 e do guia 04/2015 pela Agência Nacional de Vigilância Sanitária (ANVISA), as práticas internacionais de realização do EDF deverão ser revisadas para a obtenção de um registro de medicamento no Brasil. Portanto, este trabalho propõe um modelo de protocolo e relatório de EDF que atenda os requerimentos da legislação brasileira. Rota de degradação, condições de estresse, endpoints, percentual de degradação, balanço de massas, perfil de degradação potencial, validação analítica, identificação e 
qualificação de produtos de degradação foram discutidos ao longo deste trabalho. O modelo de protocolo e relatório apresentado atende a RDC 53/2015 em sua totalidade.

Palavras chave: estudo de degradação forçada; estudo de estresse; produto de degradação; protocolo de degradação forçada; relatório de degradação forçada.

\section{INTRODUÇÃO}

Durante o processo de fabricação e ao longo do período estipulado como prazo de validade, os medicamentos estão sujeitos a interações químicas que invariavelmente irão modificar a estrutura do fármaco nele formulado ou dos demais componentes da formulação, o que leva à formação de impurezas relacionadas, denominadas produtos de degradação. Esses produtos de degradação, assim como qualquer outra impureza presente em um medicamento, devem ser amplamente investigados com o intuito de garantir que não ultrapassem um nível que comprometa sua eficácia e segurança quanto à administração do mesmo (1). A degradação do fármaco é decorrente da ação de agentes externos, como o oxigênio, calor, luz e umidade e de sua interação com os demais componentes da formulação, os seus excipientes e dispositivos de embalagem primária utilizados para garantir a vida útil do medicamento (2).

O estudo de degradação forçada (EDF) consiste em um componente crítico no processo de desenvolvimento do medicamento, sendo considerado uma ferramenta chave para o conhecimento prévio da estabilidade do medicamento, principalmente quando se dispõe de informações limitadas sobre os potenciais produtos de degradação de um determinado insumo farmacêutico ativo ou produto acabado. Com isso, pode-se afirmar que se faz necessário realizar estudos visando avaliar a degradação do ativo não só ao fim de seu prazo de validade, mas também por meio de condições forçadas de degradação, as quais poderiam antecipar o perfil de degradação que seria obtido ao fim da vida útil do medicamento em análise $(2,3)$.

A realização do EDF em diversas condições de estresse, mais severas que as condições de estabilidade acelerada, em contato direto com a matriz na qual o medicamento é formulado, resulta em um perfil de degradação potencial cujos produtos de degradação podem estar presentes durante o estudo de estabilidade de longa duração. Os diferentes produtos obtidos deste perfil potencial de degradação serão utilizados posteriormente, no desenvolvimento e validação de uma metodologia analítica seletiva, denominada metodologia indicativa de estabilidade. Isso se deve à sua capacidade de mensurar com precisão e exatidão o insumo farmacêutico ativo e seus respectivos produtos de degradação durante o estudo de estabilidade. Além disso, o EDF irá subsidiar o desenvolvimento da formulação do produto farmacêutico em estudo, uma vez que o conhecimento químico sobre as rotas de degradação aprimora a elaboração do produto acabado, em especial, na escolha do dispositivo de embalagem primária mais efetivo para evitar ou minimizar a degradação do fármaco veiculado $(4,5)$.

Diversos guias de degradação forçada estão disponíveis para consulta em órgãos internacionais reguladores e em outras publicações sobre este tópico. Contudo, e particularmente, a falta de uma metodologia única para a condução do estudo de degradação forçada em alguns elementos essenciais, como o escopo, em que fase do estudo realizar, as condições de degradação e percentual de degradantes formados, entre outros, não são disponibilizados, o que dificulta a padronização mundial do EDF. Devido a este fato, as práticas de condução do estudo de degradação forçada adotadas por diversas companhias podem variar drasticamente, tendo um efeito significativo sobre a qualidade da metodologia analítica usada durante o estudo de estabilidade e análise de rotina de um determinado medicamento (6).

Em dezembro de 2015, a Agência Nacional de Vigilância Sanitária (Anvisa) publicou uma Resolução da Diretoria Colegiada (RDC 53/2015), que estabelece parâmetros para a notificação, identificação e qualificação de produtos de degradação (7), e seu guia associado, o Guia número 04/2015, que trata da obtenção do perfil de degradação, e identificação e qualificação de produtos de degradação em medicamentos (8). Esses documentos estão alinhados com a prática industrial e com os demais guias já descritos para a realização do EDF por outras agências internacionais. No entanto, a profundidade dos seus requisitos é expandida além dos demais, o que exige uma revisão das práticas de degradação mundialmente aplicadas para a obtenção de um registro de medicamento no mercado brasileiro e também para a manutenção dos produtos já registrados que forem submetidos a qualquer tipo de alteração pós-registro que impacte no perfil de degradação de acordo com a RDC 
171/2017 (9). O objetivo deste trabalho consistiu no desenvolvimento de um modelo de protocolo e relatório de EDF que atenda aos requerimentos preconizados na RDC 53/2015 e Guia 04/2015, publicados pela Anvisa, para auxiliar no registro de medicamento e nas futuras adequações dos produtos já registrados que fazem parte do escopo da norma brasileira, em consonância com os demais guias internacionais. Este modelo abordará todas as premissas do EDF incluindo o estabelecimento de rotas de degradação do produto acabado e os esforços para diferenciar produtos de degradação relacionados ao insumo farmacêutico ativo (IFA) dos gerados do placebo, elucidar a estrutura dos produtos de degradação, revelar os mecanismos de degradação tais como hidrólise, oxidação, termólise, fotólise do IFA e produto acabado $(10,11)$, estabelecer um método indicativo de estabilidade e um perfil de degradação potencial que envolva o perfil de degradação real do medicamento durante os estudos de estabilidade de acordo com a Resolução (RE) 01/2005 (12).

\section{MÉTODOS}

Para a elaboração de um protocolo e um relatório de EDF de medicamentos que atendam a legislação brasileira, foram utilizadas como base os requerimentos descritos na resolução e no Guia publicados pela Anvisa que tratam integralmente sobre o tema, a RDC 53/2015 e o Guia 04/2015. Adicionalmente, outras resoluções publicadas pela Anvisa foram utilizadas como suporte para atividades complementares do EDF, como a validação de método analítico descrita RDC 166/2017 (13) e a RE $01 / 2005$, que trata sobre a estabilidade de medicamentos.

\section{RESULTADOS E DISCUSSÃO}

Inicialmente, é primordial o conhecimento da melhor etapa, durante o desenvolvimento de um novo IFA e produto acabado, para a realização de EDF. O guia da Foods and Drugs Administration (FDA) recomenda que o EDF seja realizado durante a fase III do processo de submissão regulatória (14), ao passo que a Anvisa não orienta sobre a fase ideal de desenvolvimento para a realização deste estudo. Entretanto, a realização do estudo de degradação forçada conduzido no IFA em uma fase inicial, como ensaios pré-clínicos ou fase I, pode ser uma ferramenta útil referente ao tempo gasto para a identificação dos produtos de degradação e elucidação da estrutura assim como para a otimização das condições de estresse envolvidas. Uma realização precoce do EDF também pode ser útil para realizar melhorias no processo produtivo e pode ser primordial para a seleção apropriada de um método indicativo de estabilidade $(14,15)$.

O EDF deve ser realizado por meio da exposição do medicamento à agentes químicos e ou físicos (ou ambientes de estresse) capazes de promover uma degradação do IFA a partir de reações específicas de modo a prever possíveis reações que possam ocorrer com o medicamento durante o seu prazo de validade. Para isso, são utilizadas diversas condições degradantes com características reacionais distintas, para a determinação do perfil de degradação do produto acabado como por exemplo, soluções com diferentes potenciais hidrogeniônicos ( $\mathrm{pH}$ baixo, alto), agentes oxidantes, exposição à luz, exposição a elevadas temperaturas, catalisadores metálicos e exposição à umidade elevada (7). A escolha destas condições e do agente degradante deve ser consistente com a degradação do produto sem que haja formação de produtos de degradação secundários, ou seja, aqueles gerados a partir de uma primeira degradação do IFA. Segundo Reynolds e cols. (2002), após a realização de diversas entrevistas com indústrias farmacêuticas (11), a escolha do tipo de degradação vai depender da forma farmacêutica em estudo e do tipo de amostra (ativo ou produto acabado), não sendo obrigatória a realização de todas as condições descritas, o que difere do posicionamento descrito na RDC 53/2015 publicada pela Anvisa (Quadro 1). São sugeridos, aproximadamente, $10 \%$ de formação de produto de degradação com decaimento de até $90 \%$ do IFA para a determinação de um perfil de degradação satisfatório (8). Este percentual de degradação é suficiente para demonstrar que a variação não é apenas inerente à precisão do método, e garante uma precisão e exatidão adequada dos produtos de degradação. Referências na literatura podem ser utilizadas como ponto de partida para definir os endpoints para cada condição de estresse avaliada. Entretanto, esses endpoints, ou seja, o tempo final para atingir a degradação desejada, devem ser obtidos experimentalmente. Algumas empresas sugerem $30 \%$ de degradação. Mas, tal intensidade somente se justifica se a degradação de até $10 \%$ de decaimento levar à formação de apenas um produto de degradação ou à formação de produtos de degradação voláteis não identificáveis. 
Quadro 1. Protocolo para estudo de degradação forçada (Prática Global x Anvisa)

\begin{tabular}{|c|c|c|c|c|c|}
\hline \multirow{2}{*}{ Condição } & \multicolumn{2}{|c|}{ IFA (FDA, WHO, EMA) } & \multicolumn{2}{|c|}{ Medicamento (FDA, WHO, EMA) } & \multirow{2}{*}{$\begin{array}{c}\text { ANVISA } \\
\text { (Todas as formas } \\
\text { farmacêuticas) }\end{array}$} \\
\hline & Sólido & Solução Suspensão & Sólido & Solução Suspensão & \\
\hline Ácido/Base & & $\checkmark$ & & $x$ & $\checkmark$ \\
\hline Oxidação & $x$ & $\checkmark$ & $\checkmark$ & $\checkmark$ & $\checkmark$ \\
\hline Fotoestabilidade & $\checkmark$ & $x$ & $\checkmark$ & $\checkmark$ & $\checkmark$ \\
\hline Termólise & $\checkmark$ & & $\checkmark$ & $\checkmark$ & $\checkmark$ \\
\hline Termólise/ Umidade & $\checkmark$ & & $\checkmark$ & & $\checkmark$ \\
\hline Íons Metálicos & & & & & $\checkmark$ \\
\hline
\end{tabular}

Ao analisar os resultados obtidos, $100 \%$ das A intensidade de degradação deve ser rigorosamente controlada, em especial, tendo em mente a necessidade de se obter um balanço de massas próximo de $100 \%$.

O primeiro passo antes da realização do EDF propriamente dito, é a elaboração de um protocolo de estudo, definindo as instruções do ensaio, os procedimentos e os critérios de aceitação propostos para a condução do estudo.

Os critérios e procedimentos sugeridos podem ser modificados de acordo com o resultado do estudo e de possíveis limitações que podem estar associadas à técnica, as características físico-químicas da amostra e a sua própria estabilidade intrínseca. Um EDF, assim como todo protocolo de um estudo analítico, deve ser previamente revisado e aprovado, antes do início da análise. Comumente, colaboradores da mesma área, pertencentes a um nível hierárquico superior ao do autor do protocolo tais como Supervisores, Coordenadores, Gerentes, realizam as etapas de revisão e aprovação. Todo protocolo deve possuir um código de identificação interno, de acordo com a política de documentação da empresa, incluindo um controle de versões contendo o histórico de revisão para cada alteração efetuada. Este protocolo deve descrever o objetivo do estudo fornecendo uma breve descrição do objeto de avaliação e o escopo de avaliação que o inclui (16). Nesta sessão, informações referentes ao nome do medicamento, sua formulação, forma farmacêutica e sua(s) dosagem(ns) a ser(em) avaliada(s) devem ser claramente descritas, assim como o IFA em estudo, a categoria do método em avaliação (teor, impureza ou teor e impureza) e a técnica analítica empregada.

Cabe ainda descrever todos os elementos necessários para a realização do estudo de degradação forçada tais como o local de realização do estudo de degradação forçada; equipamentos a serem utilizados durante o estudo; software a ser utilizado na obtenção do método indicativo de estabilidade e na predição de produtos de degradação tóxicos; materiais analíticos; instruções especiais sobre manuseio, estabilidade e armazenamento para cada material (14).

O EDF propriamente dito inicia-se por uma extensa pesquisa bibliográfica prévia utilizando bases de dados científicas, compêndios oficiais disponíveis, buscadores diretos e no próprio Drug Master File (DMF) do fármaco. Esta pesquisa tem como objetivo mapear produtos de degradação já descritos na literatura relacionados ao fármaco e medicamento associados, impurezas relacionadas, métodos analíticos já desenvolvidos e validados, alertas de toxicidade relacionada a alguma impureza ou produto de degradação e outras informações ligadas às características físico-química do fármaco e seus produtos de degradação, tais como polimorfismo, isomeria, pKA, solubilidade, peso molecular, estrutura molecular, fórmula molecular, rotas de degradação e perfil de impurezas. É de suma importância que essas informações referentes aos produtos de degradação (identificação e toxicidade) e métodos analíticos de análise sejam disponibilizadas (preferencialmente em tabela) no protocolo de EDF, para que possam ser utilizadas como base no desenvolvimento do método indicativo de estabilidade e em possíveis mecanismos de proteção de degradação.

Antes da realização do estudo experimental de degradação forçada, é necessário que o sistema analítico seja adequadamente projetado, com manutenção em dia, calibrado e validado. Todo o pessoal que irá realizar o estudo deve possuir treinamento na técnica analítica empregada e na nas diretrizes de condução do EDF.

A metodologia analítica que será utilizada deve ser descrita inicialmente, para a separação e a quantificação dos produtos de degradação que porventura forem gerados durante a avaliação das amostras submetidas à degradação forçada. Esta metodologia pode ser anexada ao 
protocolo de EDF ou descrita no corpo do documento, conforme mencionado.

O estudo experimental de degradação forçada deve ser realizado em diversas condições para as seguintes amostras: placebo, medicamento e IFA (isolados e associados em dose fixa). Em alguns casos é interessante a avaliação do diluente ou do branco para determinadas condições de estresse, visando a identificação de possíveis sinais cromatográficos não associados aos produtos de degradação, IFA ou placebo. Os resultados encontrados com as condições de estresse, assim como seu perfil de impurezas, devem ser comparados com a amostra controle (sem exposição a qualquer condição forçada) conforme mostrado na Tabela 1.

Tabela 1. Simulação de resultados obtidos com as condições de estresse aplicadas

\begin{tabular}{|c|c|c|c|}
\hline $\begin{array}{c}\text { Condição de } \\
\text { estresse }\end{array}$ & $\begin{array}{c}\text { (\%) Área do fármaco no } \\
\text { cromatograma material } \\
\text { degradado }\end{array}$ & $\begin{array}{c}\text { (\%) Área de impurezas no } \\
\text { cromatograma material } \\
\text { degradado }\end{array}$ & $\begin{array}{c}\text { (\%) do fármaco } \\
\text { cromatograma controle }\end{array}$ \\
\hline $\begin{array}{c}\text { Amostra } \\
\text { controle }\end{array}$ & Não aplicável & Impureza 1: 0,3\% & $99,8 \%$ \\
\hline $\begin{array}{c}\text { Amostra } \\
\text { vencida }\end{array}$ & $99,1 \%$ & $\begin{array}{c}\text { Impureza 1: 0,5\% } \\
\text { Impureza 2: 0,3\% }\end{array}$ & Não aplicável \\
\hline Ácida & $88,7 \%$ & $\begin{array}{l}\text { Impureza 1: 0,3\% } \\
\text { Impureza 2: 9,0 \% }\end{array}$ & Não aplicável \\
\hline Básica & $95,4 \%$ & $\begin{array}{l}\text { Impureza 1: 0,3\% } \\
\text { Impureza 3: 3,0 } \%\end{array}$ & Não aplicável \\
\hline Metálica & $99,0 \%$ & Impureza 1: 0,3\% & Não aplicável \\
\hline 0xidativa & $91,0 \%$ & $\begin{array}{l}\text { Impureza 1: 0,7\% } \\
\text { Impureza 4: 8,5\% }\end{array}$ & Não aplicável \\
\hline Térmica & $99,3 \%$ & Impureza 1: 0,3\% & Não aplicável \\
\hline Fotolítica & $99,2 \%$ & Impureza 1: 0,3\% & Não aplicável \\
\hline Umidade & $99,1 \%$ & Impureza 1: 0,2\% & Não aplicável \\
\hline
\end{tabular}

Uma abordagem como a utilização de amostras do medicamento já expirado (validade vencida) pode ser adicionada nesta avaliação para o conhecimento do perfil de degradação real do produto, ou seja, aquele perfil que representa as impurezas no fim do estudo de estabilidade de longa duração. As condições de degradação forçada adotadas e seus endpoints podem ser inicialmente obtidos por meio de referências já publicadas, como o guia da Organização Mundial de Saúde, WHO Technical Report Series 929 - Annex 5 for registration of fixed-dose combination medicinal products (17) e no Guia 04/2015 - Guia para obtenção do perfil de degradação, e identificação e qualificação de produtos de degrada- ção em medicamentos da Anvisa (8). As condições de degradação são definidas para a obtenção de uma degradação próxima a $10 \%$. Porém, caso não seja obtida a degradação desejada, outras referências podem ser utilizadas para a determinação dos endpoints das reações de degradação. No caso de degradação superior ao valor mencionado, ou formação de produtos de degradação secundários, condições mais brandas de degradação podem ser adotadas, diminuindo o tempo de exposição ou a concentração do agente degradante utilizado, para a obtenção de um perfil de degradação primário. Na Tabela 2, são apresentadas as condições de degradação usualmente propostas na condução do EDF. 
$0.14450 / 2318-9312 \cdot v 30 . e 3 . a 2018 . p p 194-202$

Tabela 2. Condições de estresse comumente utilizadas nos estudos de degradação forçada*

\begin{tabular}{|c|c|c|}
\hline Condição de Estresse & Exposição & Tempo de Exposição \\
\hline Térmica seca & $60^{\circ} \mathrm{C}$ & $1-10$ dias \\
\hline Exposição à luz & $\begin{array}{l}2,4 \text { milhões de lux. Hora ou } \\
\text { Halogeneto metálico, mercúrio, xenônio ou ultravioleta-B }\end{array}$ & $\begin{array}{l}\text { Suficiente para a exposição de } 2,4 \\
\text { milhões de lux }\end{array}$ \\
\hline Hidrólise básica & $\mathrm{NaOH} 0,1 \mathrm{M}$ & 1- 10 dias \\
\hline Íons Metálicos & $0.05 \mathrm{M} \mathrm{Fe}^{2+}$ ou $\mathrm{Cu}^{2+}$ & 1- 10 dias \\
\hline Térmica úmida & $40^{\circ} \mathrm{C} / 75 \%$ U.R. & 10 dias \\
\hline Umidade & $75 \%$ de umidade relativa ou superior & 1- 10 dias \\
\hline 0xidação & $3 \% \mathrm{H}_{2} \mathrm{O}_{2}$ & 1- 3 horas \\
\hline Hidrólise Ácida & $\mathrm{HCl} 0,1 \mathrm{M}$ & $1-10$ dias \\
\hline
\end{tabular}

*WHO Technical Report Series 929 - Annex 5 for registration of fixed-dose combination medicinal products (17)

A metodologia analítica empregada deverá ser avaliada quanto à capacidade de ser utilizada no estudo de degradação forçada (perfil de degradação potencial) e nos estudos de estabilidade da empresa (perfil de degradação real), atuando como metodologia analítica indicativa de estabilidade. Para isso, deve ser garantida a seletividade do método por meio da separação do(s) analito(s) de interesse frente aos demais componentes da fórmula e interferentes, e avaliar a pureza espectral de cada analito a ser quantificado na metodologia aplicada. Desta forma, a técnica analítica que normalmente se apresenta como primeira escolha e que contempla tais requisitos é a cromatografia líquida de alta eficiência com detecção de arranjo de diodos (CLAE-DAD). Para a avaliação da seletividade e do perfil de impurezas, injeta-se uma solução do padrão de referência do IFA, uma solução do placebo e uma solução da amostra, todas elas na concentração de $100 \%$ conforme descrita no método. Essas 3 soluções (padrão, placebo e amostra) também são preparadas para cada condição de estresse proposta no protocolo. No caso de disponibilidade de impurezas conhecidas relacionadas ao IFA, é essencial a preparação de uma solução contaminando a amostra controle (ou placebo contaminado com IFA) com todas as impurezas disponíveis. Esta solução, conhecida como solução de identificação de impurezas, servirá como base para identificação dos picos que porventura forem detectados em cada condição de estresse avaliada. A seletividade do método ainda é desafiada pela avaliação da pureza espectral do analito de interesse, do monitoramento da resolução (sempre maior que 2,0), assimetria, número de pratos teóricos e espectro de UV dos analitos de interesse. Para critério de exclusão, devem ser avaliados os cromatogramas do branco e do placebo (controle e com agentes promotores de degradação) isolados e desconsiderar qualquer pico que for identificado nestes. Caso os critérios de aceitação definidos não sejam atingidos, mudanças na metodologia analítica devem ser realizadas para garantir que a metodologia seja indicativa de estabilidade.

Para a garantia preliminar de performance do método, além da confirmação da seletividade, é necessária a avaliação inicial de alguns parâmetros de validação analítica a fim de comprovar que o método é indicativo de estabilidade. Parâmetros como a linearidade, limite de detecção e quantificação são primordiais para a avaliação de parâmetros críticos da metodologia a ser empregada (13). A robustez do método frente às impurezas deve ser investigada, avaliando pequenas modificações nos principais parâmetros da metodologia que podem estar associados com a perda de seletividade do método. Nesta fase, é importante a avaliação de todas as alterações possíveis para adquirir o maior conhecimento da metodologia. Portanto, é necessária a avaliação de parâmetros como alterações de composição e pH da fase móvel, fluxo, temperatura da coluna, tempo de extração da amostra, volume de injeção, colunas cromatográficas de lotes diferentes, colunas cromatográficas de marcas diferentes com o mesmo revestimento de partículas, centrifugação versus filtragem, entre outros parâmetros que podem ser avaliados dependendo da metodologia analítica empregada (13).

A linearidade deve ser avaliada em triplicata em níveis de $80,90,100,110$ e $120 \%$ da concentração dos ativos na metodologia e as impurezas analisadas dos seus respectivos limites de quantificação até 120 \% do limite 
especificado de cada impureza (13). O coeficiente de correlação deve ser avaliado (comumente adotado como maior que 0,99 ), o intercepto $Y$ e a avaliação de resíduos também devem ser investigados.

Por se tratar de impurezas, é importante garantir o limite mínimo de reprodutibilidade para cada substância, bem como diferenciá-lo de possíveis ruídos do sistema cromatográfico. Para isso, avaliam-se também os limites de detecção (razão sinal ruído 3:1) e o limite de quantificação das impurezas envolvidas (razão sinal ruído 10:1). Toma-se como parâmetro de concentração de análise para realizar o estudo de linearidade na concentração próxima ao limite de quantificação do método, o valor equivalente ao limite de notificação de um produto de degradação conforme descrito nas resoluções RDC 53/2015 (7) e na RDC 166/2017 (9).

O conteúdo do relatório de estudo de degradação forçada é semelhante ao descrito no seu protocolo, adicionando os resultados obtidos durante os testes executados, a discussão elaborada destes resultados e uma conclusão sobre a efetividade da degradação (perfil de degradação) e sobre a confirmação do método indicativo de estabilidade. Caso alguma condição descrita no protocolo não seja atendida ou modificada, deve ser inserida uma justificativa para o não atendimento e para a modificação de algum parâmetro no relatório de degradação forçada.

Apresentar o cromatograma do placebo para cada condição de estresse resultante, identificando seus eventuais picos e seus possíveis produtos de degradação é fundamental. A visualização dos picos dos produtos de degradação deve ser conduzida na escala original e com uma escala ampliada em $125 \%$ para facilitar a observação do pico de impurezas. Todos os picos que aparecem exclusivamente no cromatograma do placebo devem ser excluídos na quantificação de área e não devem ser considerados como possíveis degradantes nas amostras ou no IFA. A pureza de pico do analito em estudo deve sempre ser destacada nos cromatogramas registrados. Deve-se ainda apresentar o cromatograma do IFA em cada condição de estresse e o cromatograma das amostras (em escala normal e $125 \%$ ampliada), integrando todos os picos observados que não estiverem relacionados ao placebo e ao branco, além de informar os parâmetros cromatográficos definidos na adequação do sistema do método (pratos teóricos, resolução, tempo de retenção relativo, assimetria e pureza de pico).

Todos os dados brutos das análises, como os cromatogramas obtidos diretamente do equipamento e demais tabelas geradas pelo software, devem ser compilados na forma de um relatório de dados brutos de análise.

Deve ser realizada a avaliação do balanço de massas nos cromatogramas obtidos com o medicamento e o IFA submetidos às condições de estresse, comparando-os com o cromatograma do IFA e medicamento obtido na amostra controle. Caso esse balanço de massas não alcance o valor próximo de $100 \%$, análises complementares das amostras, utilizando cromatografia líquida de alta eficiência com detector de espectrometria de massas (CLAE-MS) podem ser necessárias para a obtenção de um balanço de massa coerente com o decaimento do IFA (metodologia ortogonal).

Em caso de medicamentos em associação, o estudo com IFA isolados e associados confirma a relação do produto de degradação gerado com o respectivo IFA quando do estresse do medicamento. Os resultados obtidos devem ser descritos de acordo com a Tabela 1. A não realização de qualquer condição de estresse descrita na resolução RDC 53/2015 deve ser devidamente justificada.

Deve-se avaliar o perfil de degradação potencial obtido mapeando as principais condições que o IFA, ou medicamento, seja particularmente sensível, ou seja, quais são as condições que o determinado medicamento pode sofrer degradação considerando os processos de fabricação, embalagem, rota de degradação e influência dos excipientes que de fato contribuem para a degradação do IFA estudado, descrevendo as principais vias de degradação do IFA e suas impurezas formadas (caso conhecida).

Destacar as evidências de que o método proposto é indicativo de estabilidade e quais são os parâmetros críticos da metodologia que podem comprometer a separação do IFA e das impurezas (caso seja observada) também é importante.

A análise confirmatória do balanço de massas deve ser realizada, levando em conta diversos fatores que podem interferir em um balanço de massa positivo, tais como: variação intrínseca do procedimento de análise, artefato do método ou da degradação, possibilidade de perda de algum produto de degradação por evaporação, possibilidade de degradação do grupo cromóforo, e é fundamental para garantir a aplicabilidade do perfil de degradação potencial obtido.

Esta etapa visa suportar a confirmação de que o método é indicativo de estabilidade com base nas evidências apresentadas na discussão. Também deve-se identificar quais são os principais produtos de degradação que 
podem ser considerados marcadores durante o estudo de estabilidade, o que deve ser confirmado com a análise do medicamento com seu prazo de validade vencido submetido a condições de armazenagem de estabilidade de longa duração de acordo com a RE 01/2005 da Anvisa (12).

Após a conclusão do estudo de degradação forçada e determinação de um método indicativo de estabilidade, deve ser realizada uma validação de metodologia analítica, de acordo com o descrito na legislação vigente brasileira, RDC 166/2017 (13). Parâmetros como seletividade, linearidade, precisão (intracorrida e intercorrida), exatidão, limite de detecção, limite de quantificação, robustez e estabilidade das soluções devem ser avaliados novamente com a metodologia analítica final definida para a confirmação que o método é adequado para aquilo que destina (13).

Outros requisitos são utilizar o método indicativo de estabilidade definido durante o estudo de degradação forçada posteriormente validado, e realizar um estudo de estabilidade de acordo com a legislação vigente, RE 01/2005 para determinação do prazo de vida útil do medicamento (12).

Se ao fim do estudo de estabilidade de longa duração (perfil de degradação real) for verificada a presença de produto de degradação com concentração superior ao limite de identificação estabelecido pela RDC 53/2015 (7), o mesmo deve ser isolado e sua estrutura química identificada. Para tal, a cromatografia em coluna de sílica gel, o uso de placas preparativas de sílica gel e sílica organofílica e a cromatografia líquida preparativa devem ser utilizadas para o isolamento destes produtos de degradação. As técnicas de espectroscopia no infravermelho com transformada de Fourier (FTIR), ressonância magnética nuclear (RMN) de hidrogênio (RMN de ${ }^{1} \mathrm{H}$ ) e carbono (RMN de ${ }^{13} \mathrm{C}$ ), e a espectrometria de massas (EM) devem ser utilizadas para a identificação dos mesmos.

No caso dos estudos de estabilidade de longa duração (perfil de degradação real), deve ser indicada a presença de algum produto de degradação de concentração superior ao limite de quantificação estabelecido pela resolução RDC 53/2015 (7). O mesmo deve ser quantificado após seu isolamento e identificação. A qualificação deverá ser realizada com o uso do "Guia para a Condução de Estudos Não Clínicos de Segurança necessários ao desenvolvimento de medicamentos" (18) da Anvisa.

Deve ser proposta, inicialmente, uma metodologia analítica capaz de ser indicativa de estabilidade, descre- vendo detalhadamente o preparo de todas as soluções envolvidas assim como as condições de degradação ácida, básica, oxidante, umidade, íons metálicos e temperaturas definidas para a degradação. É importante verificar a solubilidade das amostras frente aos agentes degradantes e, se necessário, utilizar cossolvente.

Deve-se descrever o método analítico final que será utilizado durante o estudo de estabilidade, apresentando os resultados (\% de degradação) obtidos para cada condição de degradação para o placebo, IFA e medicamento. Dados brutos, como por exemplo, cromatogramas de cada condição de estresse realizada devem ser apresentados com a visualização clara de cada degradante formado, do ativo, e eventuais picos dos excipientes, identificado pelo seu tempo de retenção relativo (ou caso seja uma substância conhecida, pela sua nomenclatura). Resultados obtidos durante a validação analítica devem ser discutidos, comprovando que o método analítico utilizado possui poder indicativo de estabilidade, a partir da avaliação dos critérios de aceitação pré-definidos. Deve-se concluir, por meio dos resultados obtidos, que a metodologia é indicativa de estabilidade por apresentar seletividade e pureza espectral adequada, separando totalmente os analitos de interesse com resolução adequada, por apresentação reprodutibilidade e balanço de massas adequado para a avaliação.

\section{CONCLUSÃO}

Com a elaboração do protocolo e do relatório de estudo de degradação forçada, pode-se concluir, que o modelo proposto neste trabalho contempla os requisitos necessários para o cumprimento da RDC 53/2015 e pode ser usado para o desenvolvimento de método indicativo de estabilidade e para a predição de produtos de degradação, constituindo uma proposta válida para sistematização dos requerimentos presente na RDC 53/2015 e guia 04/2015 sobre o estudo de degradação forçada. A utilização deste modelo contribui para maior entendimento das diretrizes descritas na RDC 53/2015 por meio da disseminação das informações da norma de sua aplicação em um formato prático e palpável para aplicação experimental do estudo de degradação forçada para medicamentos sintéticos e semissintéticos evitando eventuais exigências e indeferimento de registro e pós registro de produto e é compatível com as boas práticas regulatórias e de laboratório. 


\section{REFERÊNCIAS}

1. Bajaj S, Singla D, Sakhuja N. Stability Testing of Pharmaceutical Products. J Appl Pharm Sci. 2012;2(03):129138. DOI: 10.7324/JAPS.2012.2322.

2. Alcântara FC, Rescia VC, Santos MA, Valduga CJ. Testes de Degradação Forçada para Fármacos e Medicamentos. Rev Pesq Inov Farm. 2013;5(1):38-48.

3. Blessy M, Patel RD, Prajapati PN, Agrawal YK. Development of forced degradation and stability indicating studies of drugs - A review. J Pharm Anal. 2014;4(3):159165. DOI: 10.1016/j.jpha.2013.09.003.

4. ICH Q2B guideline: validation of Analytical Procedures: methodology (revision 4), International Conference on Harmonization. 2005. [Internet]. Disponível em: $<$ http:// www.ich.org/fileadmin/Public_Web_Site/ICH_Products/ Guidelines/Quality/Q2_R1/Step4/Q2_R1_Guideline. pdf $>$.

5. Jain D, Basniwal PK. Forced degradation and impurity profiling: Recent trends in analytical perspectives. J Pharm Biomed Anal. 2013;86:11-35. DOI: 10.1016/j. jpba.2013.07.013.

6. Silva KER, Alves LDS, Soares MFR, Passos RCS, Faria AR, Rolim Neto PJ. Modelos de Avaliação da Estabilidade de Fármacos e Medicamentos para a Indústria Farmacêutica. Rev Ciênc Farm Básica Apl. 2009;30(2):129135.

7. BRASIL. Agência Nacional de Vigilância Sanitária. RDC 53, de 04 de dezembro de 2015. Estabelece parâmetros para a notificação, identificação e qualificação de produtos de degradação em medicamentos com substâncias ativas sintéticas e semissintéticas, classificados como novos, genéricos e similares, e dá outras providências. Diário Oficial da União, $\mathrm{n}^{\circ} 234$, de 8 de dezembro de 2015.

8. BRASIL. Agência Nacional de Vigilância Sanitária. Guia No 04 Versão 1, de 04 de dezembro de 2015. Guia Para Obtenção do Perfil de Degradação, e Identificação e Qualificação de Produtos de Degradação em Medicamentos. Brasília: ANVISA; 2015. Disponível em: <http://portal. anvisa.gov.br/documents/10181/2738062/Perfil+e+produtos + de + degrada $\% \mathrm{C} 3 \% \mathrm{~A} 7 \% \mathrm{C} 3 \% \mathrm{~A} 3 \mathrm{o}+\mathrm{em}+$ medicamentos.pdf/c18a4857-9a5c-4292-a1bf-07af6cad6902?version $=1.0>$.

9. BRASIL. Agência Nacional de Vigilância Sanitária. RDC 171, de 22 de agosto de 2017. Revisa a aplicabilidade da resolução da diretoria colegiada - RDC $\mathrm{n}^{\circ} 53$, de 4 de dezembro de 2015, para alterações pós-registro e os prazos desta resolução para produtos já registrados. Diário Oficial da União, nº 163, de 24 de agosto de 2017.

10. ICH guideline, Q1A (R2): Stability Testing of New Drug Substances and Products (revision 2), International Con- ference on Harmonization. Disponível em: <http://www. fda.gov/downloads/RegulatoryInformation/Guidances/ ucm128204.pdf>, 2003.

11. Reynolds DW, Facchine KL, Mullaney JF, Alsante KM, Hatajik TD, Motto MG. Available guidance and best practices for conducting forced degradation studies. Pharm Technol 2002; 26(2): 48-56.

12. BRASIL. Agência Nacional de Vigilância Sanitária. Resolução 01 de 29 de julho de 2005. Guia para realização de estudos de estabilidade. Diário Oficial da União, de 01 de agosto de 2005 .

13. BRASIL. Agência Nacional de Vigilância Sanitária. RDC 166, de 24 de julho de 2017. Dispõe sobre a validação de métodos analíticos e dá outras providências. Diário Oficial da União, n 141 , de 25 de julho 2017. Seção 1. p. 87.

14. FDA Guidance for Industry, INDs for Phase II and III Studies - Chemistry, Manufacturing, and Controls, Information, Food and Drug Administration. 2003. Disponível em: <http:/www.fda.gov/downloads/Drugs/ GuidanceComplianceRegulatoryInformation/Guidances/ ucm070567.pdf>.

15. Kats M. Forced degradation studies: regulatory consideration and implementation: stress testing studies are conducted to challenge specificity of stability-indicating and impurity- monitoring methods as part of validation protocol. BioPharm Int 2005; 18:1-7.

16. BRASIL. Agência Nacional de Vigilância Sanitária. RDC 17, de 16 de abril de 2010. Dispõe sobre as boas práticas de fabricação de medicamentos. Diário Oficial da União, no 73, de 19 de abril de 2010. Seção 1. p. 97.

17. WHO. World Health Organization. WHO technical report series 929: WHO Expert Committee on Specifications for Pharmaceutical Preparations, ed. 39, Geneva, 2005, p. 152. Disponível em: <http://apps.who.int/iris/ bitstream/10665/43157/1/WHO_TRS_929_eng.pdf $>$.

18. BRASIL. Agência Nacional de Vigilância Sanitária. Guia versão 2 de 31 de janeiro de 2013. Guia para a Condução de Estudos Não Clínicos de toxicologia e segurança farmacológica necessários ao desenvolvimento de medicamentos. Brasília: Gerência de Avaliação de Segurança e Eficácia GESEF/ANVISA. Disponível em: $<$ http://portal.anvisa.gov.br/documents/33836/2492465/ Guia + para $+\mathrm{a}+\mathrm{Condu} \% \mathrm{C} 3 \% \mathrm{~A} 7 \% \mathrm{C} 3 \% \mathrm{~A} 3 \mathrm{o}+\mathrm{de}+\mathrm{Es}-$ tudos $+\mathrm{N} \% \mathrm{C} 3 \% \mathrm{~A} 3 \mathrm{o}+\mathrm{Cl} \% \mathrm{C} 3 \% \mathrm{ADnicos}+\mathrm{de}+\mathrm{Toxi}-$ cologia + e + Seguran $\%$ C3 $\%$ A $7 \mathrm{a}+$ Farmacol $\%$ C3\%B3 gica + Necess $\%$ C3\%A 1 rios + ao + Desenvolvimento + de + Medicamentos +-+ Vers \% C $3 \%$ A 3 o $+2 /$ a8cad67c-14c8-4722-bf0f-058a3a284f75>. 